\title{
SUPPORTING INFORMATION: Spatially-Resolved Membrane Transport in a Single Cell Imaged by Second Harmonic Light Scattering
}

Mohammad Sharifian Gh. ${ }^{1, \uparrow, \ddagger}$, Michael J. Wilhelm ${ }^{1, \uparrow, *}$, Michael Moore ${ }^{2}$, and Hai-Lung Dai ${ }^{1}$

${ }_{1}^{1}$ Department of Chemistry, Temple University, 1901 N. $13^{\text {th }}$ Street, Philadelphia, PA 19122, USA.

2 Optical Science Center for Applied Research, Delaware State University, Dover, DE 19904, USA.

$\dagger$ These authors contributed equally to this work.

¥Present Address: Department of Cell Biology, University of Virginia.

* Author to whom correspondence should be addressed: (E-mail, michael.wilhelm@temple.edu).

\section{Contents:}

Human Dermal Fibroblast Cell Culture

SHG Imaging

Viability Staining Assay

\section{Human Dermal Fibroblast Cell Culture}

Human dermal fibroblast (HDF) cells were used as a primary cell culture. A vial of HDF cells was removed from liquid $\mathrm{N}_{2}$ storage and warmed for about $5 \mathrm{~min}$ in a $37^{\circ} \mathrm{C}$ water bath to be revived. A $1 \mathrm{~mL}$ aliquot of the cells was mixed with $4 \mathrm{~mL}$ of complete media and centrifuged for 5 minutes at $150 \times \mathrm{g}$. The complete media was made of $440 \mathrm{~mL}$ Dulbecco's modified Eagle medium, 1×DMEM (Cat No.: 10-013-CV, Corning) supplemented with $5 \mathrm{~mL}$ L-glutamine (Cat No.: 25-005-Cl, Corning), $5 \mathrm{~mL}$ penicillin/streptomycin (Cat No.: 30-002-Cl, Corning), and $50 \mathrm{~mL}$ fetal bovine serum (Cat No.: SH30071.03, Fisher Scientific). The media above the cell pellet was then removed, and the cells were re-suspended into $1 \mathrm{~mL}$ of complete media and mixed well. The suspended cells were then added into a tissue culture treated flask (Cat No.: 353108 , Fisher Scientific) with $5 \mathrm{~mL}, 10 \mathrm{~mL}$, or $20 \mathrm{~mL}$ of complete media for T-25, T-75, or T-150 flask, respectively. All procedures were performed in a sterile biological safety cabinet (BSC) to minimize the propensity for chemical or biological contamination. The flask was then swayed to homogenously coat the bottom surface with cells, and the cells were then checked using bright-field transmission microscopy (TM) to make sure that they were spherical and swimming freely. The flask was again swayed and placed into an incubator. The cells were grown at $37^{\circ} \mathrm{C}$ in a humidified atmosphere enriched with $5 \% \mathrm{CO}_{2}$. The health, morphology, and confluency of the adherent HDF cells were checked daily using TM.

Every 2-3 days, the cells were passaged by removing the media from the flask. Afterwards, the adherent cells were gently washed with 1×PBS (without $\mathrm{Ca}$ or $\mathrm{Mg}$ ) of $\mathrm{pH} \sim 7.4$ (Cat No.: 70011-044, Fisher Scientific). To detach the cells from the flask bottom (i.e., to transfer the cells to larger flasks and/or to freeze them), the trypsinization process was accomplished by adding $0.5 \mathrm{~mL}, 1 \mathrm{~mL}$, or $2 \mathrm{~mL}$ of $0.25 \%$ trypsin EDTA $1 \times$ (Cat No.: 25-053-Cl, Corning) into the T-25, T-75, or T-150 flask, respectively, and letting trypsin coat the bottom surface of the flask, and placing the flask back into the incubator for ca. $5 \mathrm{~min}$. The trypsinization process was then stopped by adding $2 \mathrm{~mL}, 4 \mathrm{~mL}$, or $8 \mathrm{~mL}$ of complete media into the detached 
cells (i.e., for T-25, T-75, or T-150, respectively) and repeatedly pipetting the mixture to collect all of the cells. The collected cells were then centrifuged for 5 minutes at $150 \times \mathrm{g}$, the media above the cells was removed, and the cells were then re-suspended into $1 \mathrm{~mL}$ of fresh complete media.

To prepare the HDF cells for SHG imaging experiments, the cells from specific passages were transferred from a tissue culture treated flask (e.g., T-25 flask) into a MatTek glass bottom culture dish (Part No.: P35G-1.0-14-C, MatTek Corporation) and allowed to adhere onto the glass surface for ca. 24 hours while they were covered in $2 \mathrm{~mL}$ of complete media.

\section{SHG Imaging}

All SHG imaging was performed on an LSM 780 upright multiphoton microscope (ZEISS). The laser source was a Coherent Chameleon Vision 2 mode-locked Ti:Sapphire laser (tunable from 680-1080 nm, average peak power of $3.75 \mathrm{~W}$ at $800 \mathrm{~nm}$, pulse width of $140 \mathrm{fs}$, and a repetition rate of $80 \mathrm{MHz}$ ). Experimental parameters were chosen as follows: excitation wavelength of $800 \mathrm{~nm}, 2 \%$ laser power (ca. $9.8 \mathrm{~mW}$ at the front objective), W Plan-Apochromat $20 \times$ objective lens $(\mathrm{NA}=1.0)$, pixel dwell time of 0.79 $\mu \mathrm{s} /$ pixel, image size of $1024 \times 1024$ pixels, pixel size of ca. $539 \mathrm{~nm}$, z-scan interval of ca. 3-5 $\mu \mathrm{m}$ (i.e., 3 stacks were scanned in z-axis), and a delay time of 60 seconds between consecutive images. The $400 \mathrm{~nm}$ $\mathrm{SH}$ response of $\mathrm{MG}$ was recorded in the forward propagation direction using a narrow bandpass filter $(400 \pm 10 \mathrm{~nm})$, and collected using a water condenser with $(N A=1.2)$. SHG signal was detected using a single element, non-descanned detection (NDD) PMT detector ( $Q E=22 \%)$. All data were saved in 16-bit format.

On the day of experiment, the solution of spent complete media was removed and the adherent cells on the MatTek glass bottom culture dish were gently washed with $1 \times$ PBS. Complete media was then replaced by $1 \mathrm{~mL}$ of $1 \times$ PBS to keep the cells hydrated, and the dish was placed on the microscope sample stage. The objective lens was directly focused onto the sample from above without using a cover slip. For typical experiments, $1 \mathrm{~mL}$ of $200 \mu \mathrm{M}$ MG (prepared in 1×PBS) (malachite green oxalate, Cat. No.: M9015, Sigma Aldrich) was added into the dish to achieve a final concentration of $100 \mu \mathrm{M}$ MG.

\section{Viability Staining Assay}

Following each SHG imaging experiment, the HDF cells were stained for 10 minutes with $10 \mu \mathrm{M} \mathrm{PI}$ and $5 \mu \mathrm{M}$ SYTO 9 (Molecular Probes ${ }^{\mathrm{TM}}$ ). Both dyes were prepared in $1 \times$ PBS solution. In order to avoid effects of phototoxicity and photobleaching, the samples were maintained under dark conditions prior to image acquisition. Single-photon fluorescence images were then acquired for both PI (561 nm excitation / $620 \mathrm{~nm}$ detection) and SYTO 9 (488 nm excitation / $535 \mathrm{~nm}$ detection). 